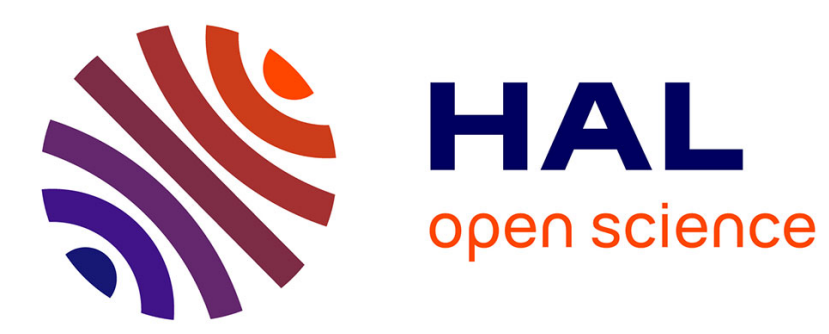

\title{
Verified Abstract Interpretation Techniques for Disassembling Low-level Self-modifying Code
}

\author{
Sandrine Blazy, Vincent Laporte, David Pichardie
}

\section{To cite this version:}

Sandrine Blazy, Vincent Laporte, David Pichardie. Verified Abstract Interpretation Techniques for Disassembling Low-level Self-modifying Code. Journal of Automated Reasoning, 2016, 56 (3), pp.26. 10.1007/s10817-015-9359-8 . hal-01243700

\section{HAL Id: hal-01243700 \\ https://hal.inria.fr/hal-01243700}

Submitted on 27 May 2016

HAL is a multi-disciplinary open access archive for the deposit and dissemination of scientific research documents, whether they are published or not. The documents may come from teaching and research institutions in France or abroad, or from public or private research centers.
L'archive ouverte pluridisciplinaire HAL, est destinée au dépôt et à la diffusion de documents scientifiques de niveau recherche, publiés ou non, émanant des établissements d'enseignement et de recherche français ou étrangers, des laboratoires publics ou privés. 


\title{
Verified Abstract Interpretation Techniques for Disassembling Low-level Self-modifying Code
}

\author{
Sandrine Blazy · Vincent Laporte . \\ David Pichardie
}

Received: date / Accepted: date

\begin{abstract}
Static analysis of binary code is challenging for several reasons. In particular, standard static analysis techniques operate over control-flow graphs, which are not available when dealing with self-modifying programs which can modify their own code at runtime. We formalize in the Coq proof assistant some key abstract interpretation techniques that automatically extract memory safety properties from binary code. Our analyzer is formally proved correct and has been run on several self-modifying challenges, provided by Cai et al. in their PLDI 2007 article.
\end{abstract}

Keywords Coq · abstract interpretation · low-level programming language

Publication history: this article is a revised and extended version of the paper "Verified Abstract Interpretation Techniques for Disassembling Low-level Self-modifying Code" published in the ITP 2014 conference proceedings.

\section{Introduction}

Abstract interpretation [16] provides advanced static analysis techniques with strong semantic foundations. It has been applied on a large variety of programming languages. Still, specific care is required when adapting these techniques

This work was supported by Agence Nationale de la Recherche, grant number ANR-11-INSE003 Verasco.

\author{
S.Blazy \\ Université Rennes 1 - IRISA - Inria, Campus de Beaulieu, Rennes, France \\ E-mail: Sandrine.Blazy@irisa.fr \\ V.Laporte \\ Université Rennes 1 - IRISA - Inria, Campus de Beaulieu, Rennes, France \\ E-mail: Vincent.Laporte@irisa.fr \\ D.Pichardie \\ ENS Rennes - IRISA - Inria, Campus de Beaulieu, Rennes, France \\ E-mail: David.Pichardie@ens-rennes.fr
}


to low-level code, specially when the program to be analyzed comes in the form of a sequence of bits and must first be disassembled. Disassembling is the process of translating a program from a machine friendly binary format to a textual representation of its instructions. It requires to decode the instructions (i.e., understand which instruction is represented by each particular bit pattern) but also to precisely locate the instructions in memory. Indeed instructions may be interleaved with data or arbitrary padding. Moreover once encoded, instructions may have various byte sizes and may not be well aligned in memory, so that a single byte may belong to several instructions.

To thwart the problem of locating the instructions in a program, one must follow its control-flow. However, this task is not easy because of the indirect jumps, whose targets are unknown until runtime. A static analysis needs to predict with enough precision, given an expression denoting a jump target, the values it may evaluate to.

In addition, instructions may be produced at runtime, as a result of the very execution of the program. A simple example is the modification of some operands (e.g., registers) of existing instructions. Another example is the creation of new sequences of instructions in existing code. Such programs are called self-modifying programs; they are commonly used in security as an obfuscation technique (e.g., to protect the intellectual property of the program authors, to increase the stealth of malware) [33], as well as in just-in-time compilation and in operating systems (mainly for improving performances).

Analyzing a binary code is mandatory when this code is the only available part of a software. Because the instructions of a self-modifying program are not the instructions that will be executed, most of standard reverse engineering tools (e.g., the IDA Pro disassembler and debugger, a de facto standard for the analysis of binary code) cannot disassemble and analyze self-modifying programs. In order to disassemble and analyze such programs, one must very precisely understand which instructions are written and where. And for all programs, one must check every single memory write to decide whether it modifies the program code.

As the real code of a self-modifying program is hidden and varies over time, self-modifying programs are also beyond the scope of the vast majority of formal semantics of programming languages. Indeed a prerequisite in such semantics is the isolation and the non-modification of code in memory. Turning to verified static analyses, they operate over toy languages $[8,27]$ or more recently over realistic C-like languages $[32,6]$, but they assume that the control-flow graph is extracted by a preliminary step, and thus they do not encompass techniques devoted to self-modifying code.

In this paper, we formalize with the Coq proof assistant key static analysis techniques to predict the possible targets of the computed jumps and make precise which instructions alter the code and how, while ensuring that the other instructions do not modify the program. Our static analysis techniques rely on two main components classically used in abstract interpretation, abstract domains and fixpoint iterators, that we detail in this paper. 
Our formalization effort is divided in three parts. Firstly, we formalize a small binary language in which code is handled as regular mutable data. Secondly, we formalize and prove correct an abstract interpreter that takes as input an initial memory state, computes an over-approximation of the reachable states that may be generated during the program execution, and then checks that all reachable states maintain memory safety. Finally, we extract from our formalization an executable OCaml tool that we run on several self-modifying challenges, provided by Cai et al. [11].

The article makes the following contributions.

- We push further the limit in terms of verified static analysis by tackling the specific challenge of binary self-modifying programs, such as fixpoint iteration without control-flow graph and simple trace partitioning [22].

- We provide a complementary approach to [11] by automatically inferring the required state invariants that enforce memory safety. Indeed, the axiomatic semantics of [11] requires programs to be manually annotated with invariants written in a specific program logic.

The remainder of this article is organized as follows. First, Section 2 briefly introduces the static analysis techniques we formalized. Then, Section 3 defines the semantics of our low-level language. Section 4 details our abstract interpreter. Section 5 describes some improvements that we made to the abstract interpreter, as well as the experimental evaluation of our implementation. We finish this article by a discussion of related work in section Section 6 , followed by future work and conclusions in section Section 7.

Availability The Coq development underlying this article can be consulted on-line at http://www.irisa.fr/celtique/ext/smc.

Notations Option types are used to represent potential failures. For functions returning "option" types, $\lfloor x\rfloor$ (read: "some $x ")$ corresponds to success with return value $x$, and $\varnothing$ (read: "none") corresponds to failure. In the context of abstract interpretation, where abstract values represent sets of concrete values, it is often convenient to distinguish two interpretations of such an option type: the absence of a definite value may be interpreted as the empty set or as the full set. Therefore, we introduce two different option types, botlift A and toplift A, abbreviated respectively $\mathrm{A}+\perp$ and $\mathrm{A}+\mathrm{T}$ :

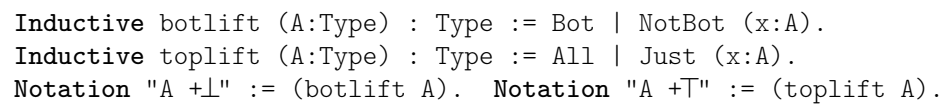

These types are equipped with a monad structure; hence we use standard operators bind, lift and notation do $\mathrm{a} \leftarrow \mathrm{m}$; b. We note $\perp$ for Bot, $T$ for Top, and overload the notation $\lfloor x\rfloor$ for NotBot $\mathrm{x}$ and Just $\mathrm{x}$.

\section{Disassembling by Abstract Interpretation}

We now present the main principles of our analysis on the program shown in Figure 1. It is printed as a sequence of bytes (on the extreme left) as well 


\begin{tabular}{ccc} 
Initial program & Possible final program & Initial assembly listing \\
\hline 07000607 & 07000607 & $0:$ cmp R6, R7 \\
03000000 & 03000000 & $1:$ gotoLE 5 \\
00000005 & 00000004 & $2:$ \\
00000000 & 00000000 & $3:$ halt R0 \\
00000100 & 00000100 & $5:$ halt R1 \\
09000000 & 09000000 & $6:$ Ro 4 \\
00000004 & 00000004 & $7:$ cst $2 \rightarrow$ R2 \\
09000002 & 09000002 & $8:$ \\
00000002 & 00000002 & $9:$ store R0 $\rightarrow * R 2$ \\
05000002 & 05000002 & $10:$ goto 1 \\
04000000 & 04000000 & $11:$ \\
00000001 & 00000001 &
\end{tabular}

Figure 1 A self-modifying program: as a byte sequence (left); after some execution steps (middle); assembly source (right).

as under a disassembled form (on the extreme right) for readability purposes. This program, as we will see, is self-modifying, so these bytes correspond to the initial content of the memory from addresses 0 to 11 . The remainder of the memory (addresses in $\left[-2^{31} ;-1\right] \cup\left[12 ; 2^{31}-1\right]$ ), as well as the content of the registers, is unknown and can be regarded as the program input.

All our example programs target a machine operating over a low-level memory made of $2^{32}$ cells, eight registers, and flags - boolean registers that are set by comparison instructions. Each memory cell or register stores a 32 bits integer value, that may be used as an address in the memory. Programs are stored as regular data in the memory; their execution starts from address zero.

Nevertheless, throughout this paper we write the programs using the following custom syntax. The instruction cst $\mathrm{v} \rightarrow \mathrm{r}$ loads register $r$ with the given value v. The instruction cmp $r, r$ ' denotes the comparison of the contents of registers $r$ and $r$ '. The instruction gotoLE $d$ is a conditional jump to $d$, it is taken if in the previous comparison the content of $r$ ' was less than or equal to the one of $r$; goto $d$ is an unconditional jump to $d$. The instructions load $* r \rightarrow r$ ' and store $r^{\prime} \rightarrow * r$ denote accesses to memory at the address given in register $r$; and halt $r$ halts the machine with as final value the content of register $r$.

The programming language we consider is inspired from x 86 assembly; notably instructions have variable size (one or two bytes, e.g., the length of the instruction gotoLE 5 stored at line 1 is two bytes, the byte 03000000 for goto and one byte for 5) and conditional jumps rely on flags. In this setting, a program is no more than an initial memory state, and a program point is simply the address of the next instruction to execute.

In order to understand the behavior of this program, one can follow its code as it is executed starting from the entry point (byte 0 ). The first instruction cmp R6, R7 compares the (statically unknown) content of two registers. This comparison modifies only the states of the flags. Then, the gotoLE 5 instruction is executed and, depending on the outcome of this comparison, the execution proceeds either to the following instruction (stored at byte 3 ), or from byte 5 . 
Since the analysis cannot predict which branch will be taken, both branches must be analyzed.

Executing the block from byte 5 will modify the byte 2 belonging to the gotoLE instruction (highlighted in Figure 1); more precisely it will change the jump destination from 5 to 4 : the store RO $\rightarrow *$ R2 instruction writes the content of register Ro (namely 4 ) in memory at the address given in register R2 (namely 2 ). Notice that a program may directly read from or write to any memory cell: we assume that there is no protection mechanism as provided by usual operating systems. After the modification is performed, the execution jumps back to the modified instruction, jumps to byte 4 then halts, with final value the content of register R1.

This example highlights that the code of a program (or its control-flow graph) is not necessarily a static property of this program: it may vary as the program runs. To correctly analyze such a program, one must discover, during the fixpoint iteration, the two possible states of the goto instruction at program points 1 and 2 and its two possible targets (i.e., 4 and 5). More specially, we need at least to know, for each program point (i.e., memory location), which instructions may be decoded from there when the execution reaches this point. This in turn requires to know what are the values that the program operates on. We therefore devise a value analysis that computes, for each reachable program point (i.e., in a flow sensitive way) an over-approximation of the content of the memory and the registers, and the state of the flags, when the execution reaches that point.

The analysis relies on a numeric abstract domain $N^{\sharp}$ that provides a representation for sets of machine integers and abstract arithmetic operations. $\gamma_{\mathrm{N}} \in \mathrm{N}^{\sharp} \rightarrow \mathcal{P}$ (int) denotes the associated concretization function. Relying on such a numeric domain, one can build abstract transformers. They model the execution of each instruction over an abstract memory that maps locations (i.e., memory addresses ${ }^{1}$ and registers) to abstract numeric values. An abstract state is then a mapping that attaches such an abstract memory to each program point of the program, and thus belongs to addr $\rightarrow\left((\right.$ addr + reg $\left.) \rightarrow N^{\sharp}\right)$.

To perform one abstract execution step, from a program point pp and an abstract memory state $\mathrm{m}^{\sharp}$ that is attached to $\mathrm{pp}$, we first enumerate all instructions that may be decoded from the set $\gamma_{N}\left(\mathrm{~m}^{\sharp}(\mathrm{pp})\right)$. Then for each of such instructions, we apply the matching abstract transformer. This yields a new set of successor states whose program points are dynamically discovered during the fixpoint iteration.

The abstract interpretation of a whole program iteratively builds an approximation executing all reachable instructions until nothing new is learned. This iterative process may not terminate, since there might be infinite increasing chains in the abstract search space. As usual in abstract interpretation, we accelerate the iteration using widening operations [16]. Once a stable approximation is finally reached, an approximation of the program listing or control-flow graph can be produced.

1 Type addr is a synonym of int, the type of machine integers. 

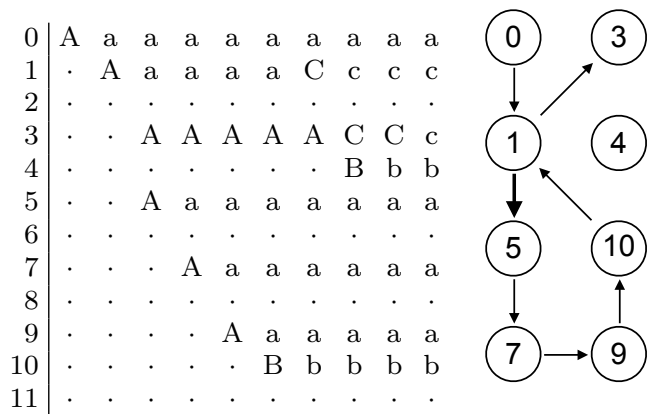

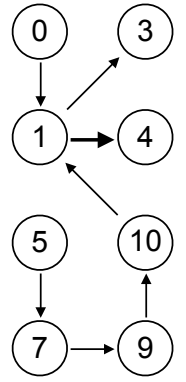

b

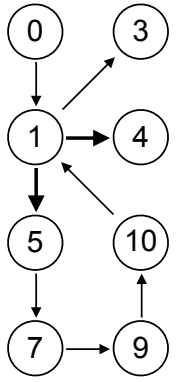

C

Figure 2 Iterative fixpoint computation

To illustrate this process, Figure 2 shows how the analysis of the program from Figure 1 proceeds. We do not expose a whole abstract memory but only the underlying control-flow graph it represents. On this specific example, three different graphs are encountered during the analysis. For each program point pp, we represent a node with same name and link it with all the possible successor nodes according to the decoding of the set $\gamma_{N}\left(m^{\sharp}(p p)\right)$. The array shows the construction of the fixpoint: each line represents a program point and the columns represent the iterations of the analysis. In each array cell lies the name of the control-flow graph representing the abstract memory for the given program point during the given iteration; a dot stands for an unreachable program point. The array cells whose content is in upper case highlight the program points that need to be analyzed: they are the worklist.

Initially, at iteration 0 , only program point 0 is known to be reachable and the memory is known to exactly contain the program denoted by the first control-flow graph (called $a$ in Figure 2 and corresponding to the initial program of Figure 1). The only successor of point 0 is point 1 and it is updated at the next iteration. After a few iterations, point 9 is reached and the abstract control-flow graph $a$ is updated into the control-flow graph $b$ that is propagated to point 10. This control-flow graph corresponds to the possible final program of Figure 1, where program-point 5 became unreachable. At the next iteration, program point 1 (i.e., the loop condition) is reached again and the control-flow graph $b$ is updated into the control-flow graph $c$ that correspond to the union of the two previous control-flow graphs. After a few more iterations, the process converges.

In addition to a control-flow graph or an assembly listing, more properties can be deduced from the analysis result. We can prove safety properties about the analyzed program, like the fact that its execution is never stuck.

The analysis produces an over-approximation of the set of reachable states. In particular, a superset of the reachable program points is computed, and for each of these program points, an over-approximation of the memory state when the execution reaches this program point is available. Thus we can check that for every program point that may be reached, the next execution step 


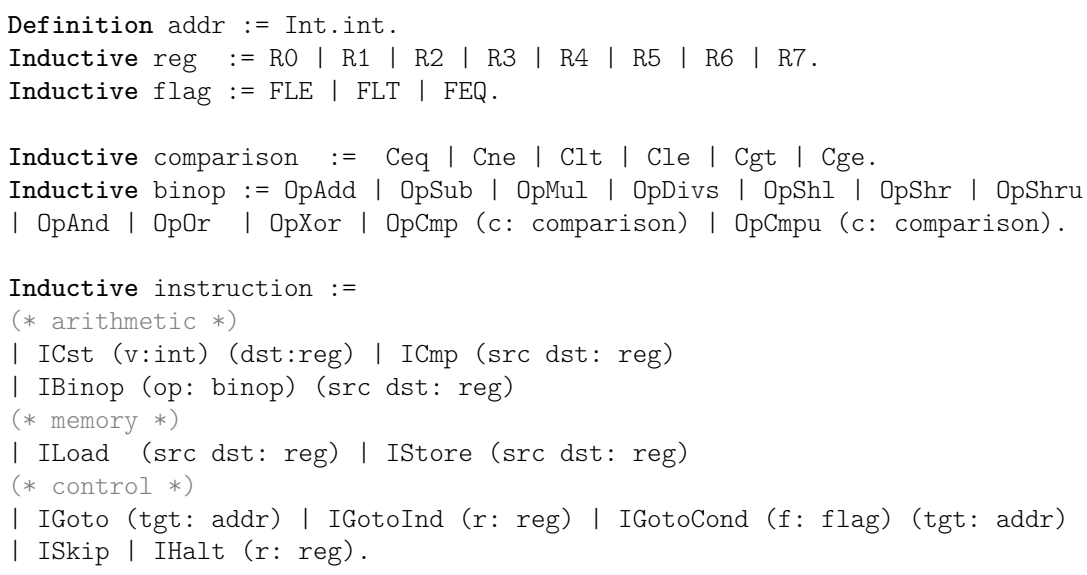

Figure 3 Abstract syntax of our low-level language

from this point cannot be stuck. This verification procedure is formally verified, as described in the following section.

\section{Semantics of our Binary Language}

This section defines the abstract syntax and semantics of the low-level language our static analyzer operates over. The semantics uses a decoding function from binary code to our low-level language. The semantics is presented as a small-step operational semantics that can observe self-modifying programs.

\subsection{Abstract Syntax}

The programming language in which are written the programs to analyze is formalized using the abstract syntax shown on Figure 3. In the Coq formalization, the abstract syntax is presented as inductive data types. Machine integers (type int) are those of the CompCert library Int of 32 bits machine integers [5]. The eight registers of our language are called $\mathrm{Ro}, \ldots \mathrm{R} 7$ and there are three register flags called FLE (for "less or equal" comparisons), FLT (for "less than" comparisons) and FEQ (for "equality" comparisons).

Instructions are either arithmetic expressions, or memory accesses or controlflow instructions. Instructions for accessing memory are ILoad and IStore; their operands are registers. So as to keep the language simple, memory accesses are limited to these two instructions: the other instructions, which are described next, only operate on registers. Arithmetic expressions consist of integer constants, signed comparisons and binary operations. Control-flow instructions consist of unconditional and conditional jump instructions, the empty instruction ISkip and the IHalt instruction which halts the program execution. For 
unconditional jumps, we distinguish register-indirect jumps (IGotoInd $r$ instructions, where $r$ is a register) from other jumps (i.e., absolute jumps, written as IGoto $\mathrm{v}$, where $\mathrm{v}$ is a literal constant address).

In a binary language, there is no distinction between code and data: a value stored in memory can be interpreted either as representing data or as encoding instructions. So as to model a binary language, we first introduce a decoding function called dec. Its type is (addr $\rightarrow$ int $) \rightarrow p p \rightarrow$ option(instruction $\times$ nat). Given a memory mem of type addr $\rightarrow$ int (i.e., a function from addresses to values) and an address pp of type addr, this function yields the instruction stored at this address along with its byte size (so as to know where the next instruction begins). This size is of type nat, the Coq type for natural numbers. Since not all integer sequences are valid encodings, this decoding may fail (hence the option type). In order to be able to conveniently write programs, there is also a matching encoding function called enc, whose type is instruction $\rightarrow$ list int. However the development does not depend on it at all: properties are stated in terms of already encoded programs.

The decoding function is defined in Figure 4. The binary decoding of a sequence of bytes stored in memory $m$ at program point $\mathrm{pp}$ is written (dec $\mathrm{m} \mathrm{pp}$ ). A successful decoding yields a pair ( $i, s z)$, where sz is the size of $i$, the instruction stored at address $\mathrm{pp}$ in $\mathrm{m}^{2}$.

The binary format of instructions is arbitrary and has little impact on the design of the analyzer. We rely on the fact that the encoding length can be inferred from the first byte of any encoded instruction ${ }^{3}$. The self-modifying programs that we consider rely on the particular encoding that we chose. This encoding works as follows. Instructions that hold a value (e.g., IGoto 5) require two bytes: the value occupies the second byte; other instructions require one byte. The first byte is made of four fields of one octet each: the decoding of this first byte first extracts the content of each field using Euclidean divisions (performed by the split_instruction function). The first field (typ) corresponds to the constructor of the instruction data type. From its value, one can deduce the size of the instruction and how to interpret the next fields. The second field ( $f \mathrm{~g}$ ) holds a flag (only used in the IGotoCond instruction). The third and fourth fields hold respectively the source and destination registers. Depending on the instruction, none, both or only one of them may be relevant. Unused fields always have the value zero. This encoding is very sparse: many byte sequences do not represent any valid instruction. Moreover, in the decoding function, errors are propagated by the bind operator of the error monad, written do $a \leftarrow m ; b$

\footnotetext{
2 The size cannot be deduced from the instruction as: 1 . the encoding function is not known; and 2. they may be several encodings, of various sizes, for a single instruction.

3 This is not the case, for instance, of the encoding of x86 instructions, that may begin with an arbitrary number of one-byte prefixes
} 


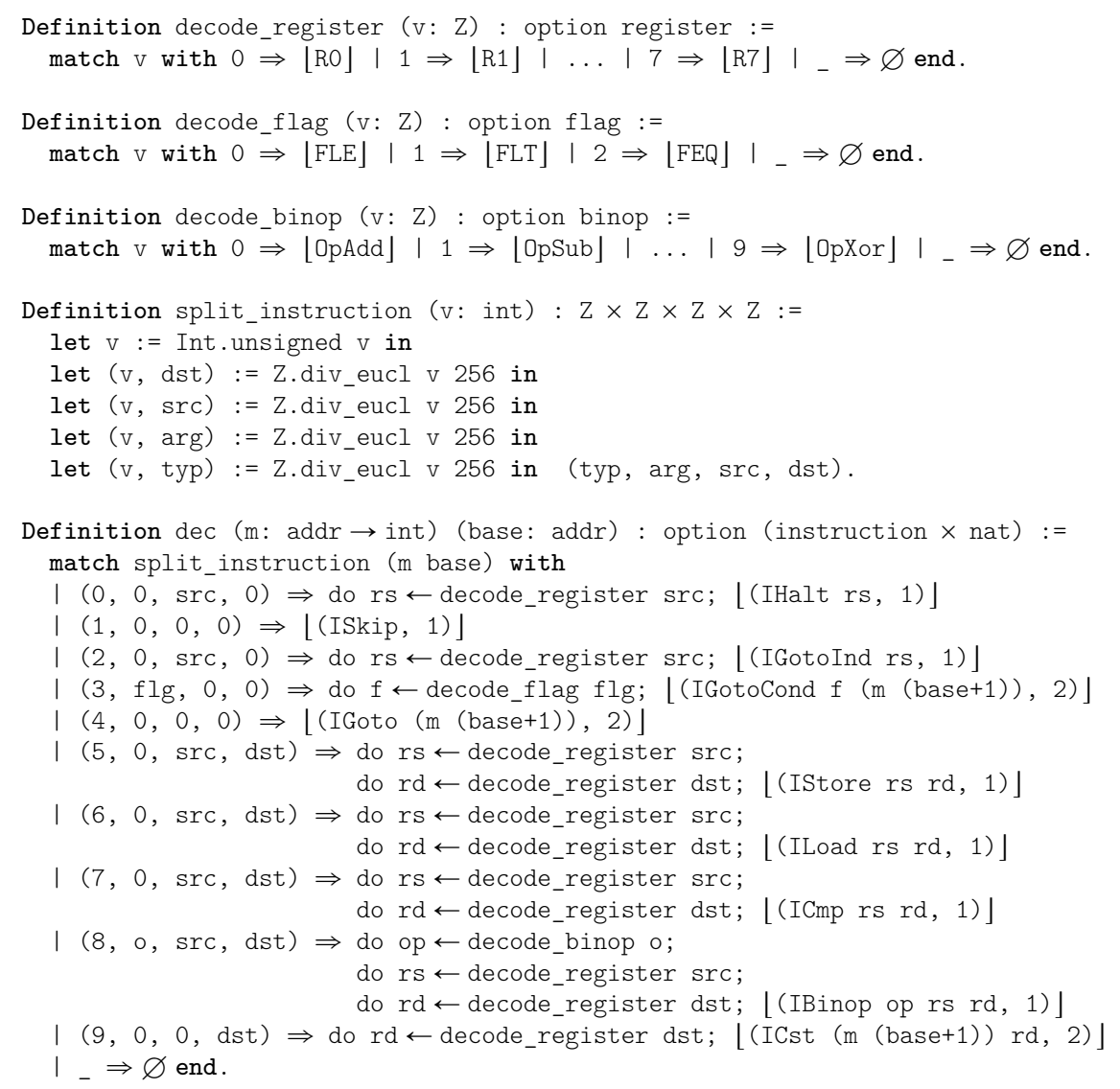

Figure 4 Decoding binary code

\subsection{Semantics}

The language semantics is given as a small-step transition relation between machine states. A machine state may be $\langle p p, f, r, m\rangle$ where pp is the current program point (address of the next instruction to be executed), $f$ is the current flag state, $r$ is the current register state, and $m$ is the current memory. Such a tuple is called a machine configuration (type machine_config). Otherwise, a machine state is $\lceil\mathrm{v}\rceil$, meaning that the program stopped returning the value $\mathrm{v}$. Values are machine integers (type int).

The semantics is defined in Figure 5 as a set of rules of the following shape:

$$
\frac{\operatorname{dec} m p p=\lfloor(i, s z)\rfloor}{\langle p p, f, r, m\rangle m\left\langle\left\langle p^{\prime}, f^{\prime}, r^{\prime}, m^{\prime}\right\rangle\right.} .
$$

The premise states that decoding the bytes in memory $\mathrm{m}$ from address $\mathrm{pp}$ yields the instruction $i$ whose size in memory is sz. Then each rule describes 


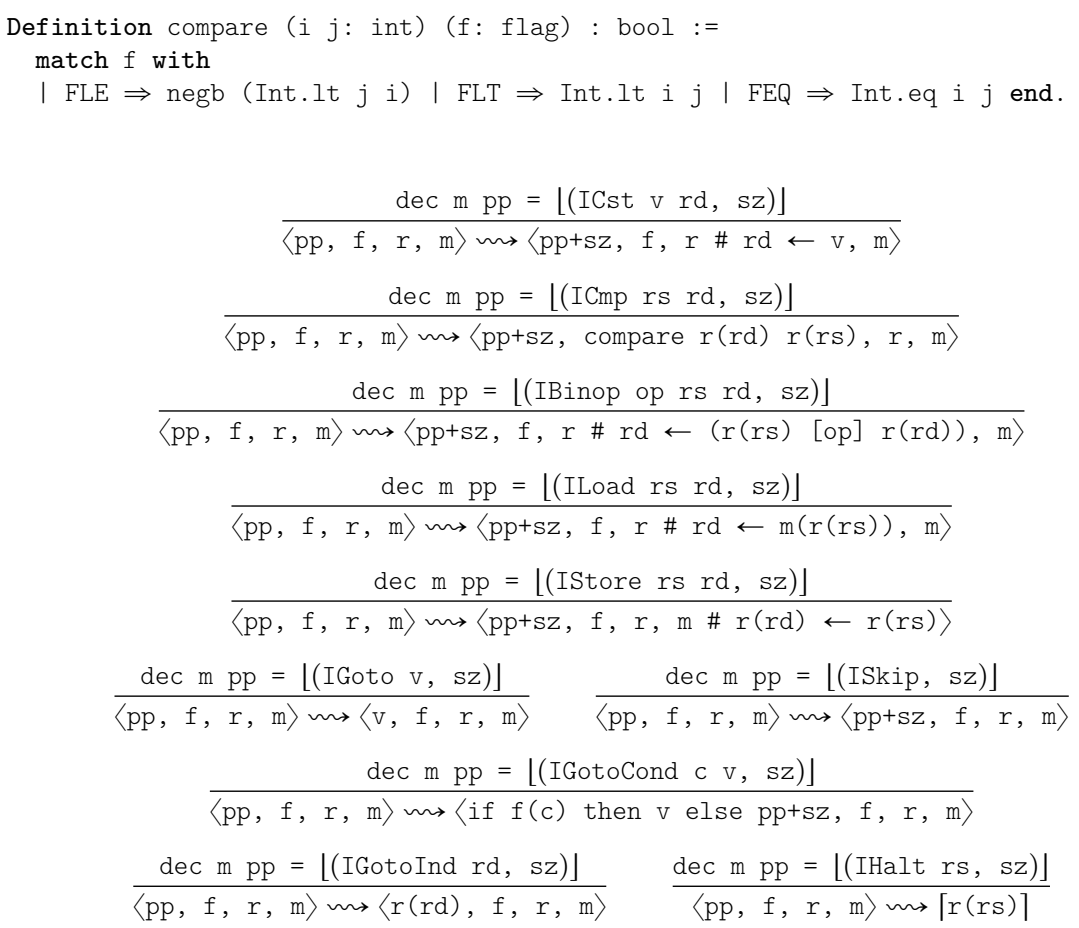

Figure 5 Concrete semantics

how to execute a particular instruction at program point $\mathrm{pp}$ in memory $\mathrm{m}$ with flag state $f$ and register state $r$. In each case, most of the state is kept unchanged. Instructions that are not branching proceed their execution at program point $\mathrm{pp}+\mathrm{sz}$ (since sz is the size of this instruction once encoded). In the rules, the notation $\mathrm{s} \# \mathrm{id} \leftarrow \mathrm{v}$ stands for the update of state $\mathrm{s}$ with a new value $\mathrm{v}$ of register or memory cell id.

Instruction ICst $\mathrm{v}$ rd updates destination register $\mathrm{rd}$ with value $\mathrm{v}$. Instruction ICmp rs rd updates the flag state according to the comparison (compare) of the values held by the two involved registers. Instruction IBinop op rs rd applies the denotation [op] of the given binary operator op to the contents $r(r s)$ and $r(r d)$ of registers $r s$ and $r d$. Then, it updates the state of register $r d$ : in $r$, the new value of $r d$ thus becomes $r(r s)$ [op] $r(r d)$. Instruction ILoad rs rd updates register $r d$ with the value $\mathrm{m}(\mathrm{r}(\mathrm{rs}))$ found in memory at the address given in register rs. Instruction IStore rs rd updates the memory at the address given in register $r d$ with the value given in register $r s$.

Instruction IGoto $\mathrm{v}$ sets the program point to $\mathrm{v}$. Instruction ISkip does nothing: execution proceeds at next program point. Conditional jump instruction IGotoCond c v jumps to address $v$ or falls through to pp+sz depending on the current state of flag c. Indirect jump instruction IGotoInd rd proceeds at the pro- 
gram point found in register rd. Instruction IHalt rs terminates the execution, returning the content of register rs.

Finally, we define the semantics $\llbracket \mathrm{P} \rrbracket$ of a program $\mathrm{P}$ as the set of states $s$ that are reachable from an initial state $\langle 0, f, r, P\rangle$, with current program point zero and memory P (where $\iota^{\star}$ denotes the reflexive-transitive closure of the small-step relation):

$$
\llbracket \mathrm{P} \rrbracket=\left\{s \mid \exists \mathrm{f} r,\langle 0, \mathrm{f}, \mathrm{r}, \mathrm{P}\rangle \mathrm{m}^{\star} s\right\} .
$$

Notice that the program P belongs to the state: it is initially known, but can be modified as the execution goes on.

\section{Abstract Interpreter}

The static analyzer is specified, programmed and proved correct using the Coq proof assistant. This involves several steps that are described in this section: designing abstract domains and abstract semantics, as well as writing a fixpoint iterator, and lastly stating and proving soundness properties about the results of the static analysis.

In order to analyze programs, we build an abstract interpreter, i.e., an executable semantics that operates over abstract elements, each of them representing many concrete machine configurations. Such an abstract domain provides operators that model basic concrete operations: read a value from a register, store some value at some address in memory, and so on. The static analyzer then computes a fixpoint within the abstract domain, that overapproximates all reachable states of the analyzed program. We first describe our abstract domain before we head to the abstract semantics and fixpoint computation.

\subsection{Abstract Domains}

Our abstract interpreter operates over an abstract memory domain. This abstract domain handles a (simplified) lattice structure, plus some abstract transformers that we describe below. It is parameterized by a numeric abstract domain that abstracts numerical values. The same notion of lattice structure is used in both signatures. We now describe these different signatures.

Weak Lattice. Each abstract domain handles a set of lattice operators that are convenient for programming abstract transformers and performing fixpoint computation. Figure 6 presents the type class weak_lattice. It is parameterized by a carrier type $\mathrm{A}$ and handles an order test leb, a top element, a join operator and a widening operator. The class only contains the operator signatures and not their specifications. 


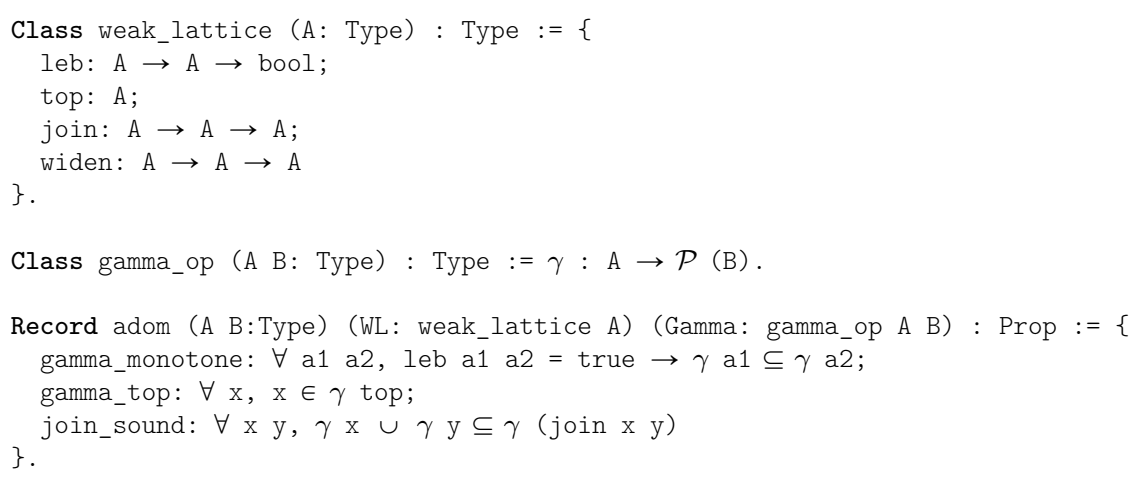

Figure 6 Signature of weak lattices and concretizations

In the same figure, we provide the record signature adom that contains soundness specifications for leb, top and join. We rely on an abstract interpretation methodology that only manipulates concretization functions, instead of full Galois connections (see [9] for a discussion about this design choice). The concretization operator $\gamma$ transforms an abstract element into its counterpart concrete property (i.e., a set of concrete elements). A concrete property $P \in \mathcal{P}(A)$ is conservatively (over-)approximated by an abstract element $p^{\sharp}$ if $P \subseteq \gamma\left(p^{\sharp}\right)$

A concretization operator is given a dedicated type class signature. This design choice allows us to overload the $\gamma$ notation and let the type class inference mechanism automatically infer which instance of gamma_op must be considered each time we write $\gamma$. The same facility is used for type weak_lattice. As a consequence, the weak lattice wL and the gamma operator Gamma are implicitly considered in the fields of record adom.

Note at last, that we do not require any property for the widening operator. It is used during fixpoint computation to speedup convergence but we do not prove termination of this iterative process and validate a posteriori the correctness of the obtained limit.

Numeric Abstract Domain. The heart of our abstract interpreter performs numeric abstraction in order to infer numeric properties on the memory content. Figure 7 gives the signature of numeric abstract domains ab_machine_int. In addition to the previous weak_lattice, gamma_op and adom components, the record handles three operators concretize, const_int and forward_int_binop, together with their specifications.

The operator concretize transforms an abstract numeric value into a finite set of machine integers it represents. This set is always finite but can be very large. The type fint_set contains a special constructor that implicitly represents all integers. We can rely on it when we do not want to enumerate the elements of a too large set. Note that this type is equipped with its own gamma_op instantiation and we implicitly use it in the specification concretize_correct. 


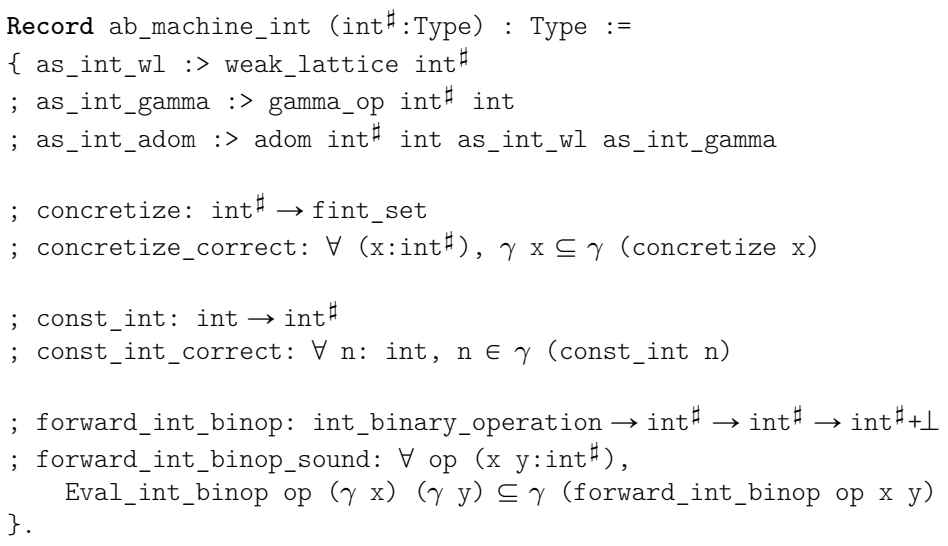

Figure 7 Signature of abstract numeric domains

This operator is necessary when we need to concretize a set of memory cells that may be targeted by a memory load or store.

The other operators are standard forward abstract transformers. The operator const_int returns the best abstraction for a constant and forward_int_binop approximates the denotation [op] (see Section 3) of a binary operator; the Eval_int_binop function that appears in its specification lifts this denotation to sets of machine integers.

We provide two numeric domains that instantiate this interface: intervals with congruence information [1] and finite sets. This part of the development is described in [6] and we have made our formal development sufficiently modular to benefit from future improvements in the Verasco project [20].

Memory Abstract Domain. An abstract memory domain is a carrier type along with some primitive operators whose signatures are given in Figure 8. The carrier type ab_mc is equipped with a lattice structure. An object of this type represents a set of triples flag-state $\times$ register-state $\times$ memory, as described by the primitive gamma. Such a triple ultimately represents any machine configuration with matching components at any program point (see gamma_to_mc).

A memory domain can be queried for the values stored in some register (var) or at some known memory address (load_single); these operators return an abstract numeric value. Other operators enable us to alter an abstract state, like assign that sets the contents of a register to a given abstract numeric value, and store_single that similarly updates the memory at a given address.

The operator compare updates the abstract counterpart of the flag state when two given registers are compared. We can also use the operator assume when we know the boolean value of a flag. This operator is a reduction. It is always sound to return the same abstract state as the first argument, but a more precise information may allow to gain precious information when reaching a conditional branch. The operator init is used when initializing the abstract 


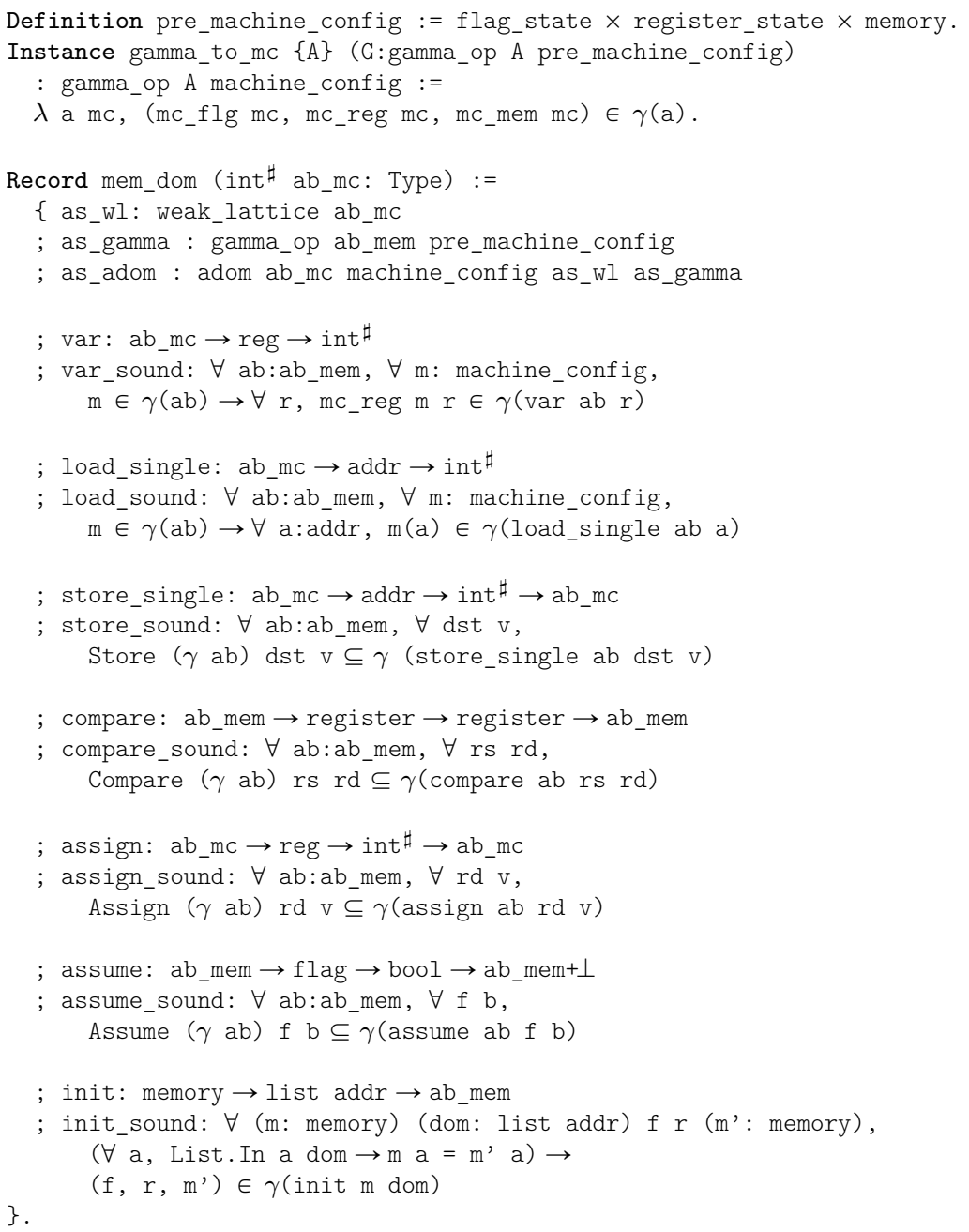

Figure 8 Signature of abstract memory domains

interpreter with an abstraction of the initial memory. Part of the initial memory is exactly known: the initial program text, static data and so on. Therefore the init operator gets a list dom of addresses and a function $\mathrm{m}$ that gives the values of the actual initial memory m' at these addresses.

All these operators obey some specifications. As an example, the load_sound property states that given a concrete state $\mathrm{m}$ in the concretization of an abstract state $a b$, the concrete value stored at any address a in $m$ is over-approximated by the abstract value returned by the matching abstract load. The $\gamma$ symbol is overloaded through the use of type classes: its first occurrence refers to the concretization from the abstract memory domain (the gamma field of record 


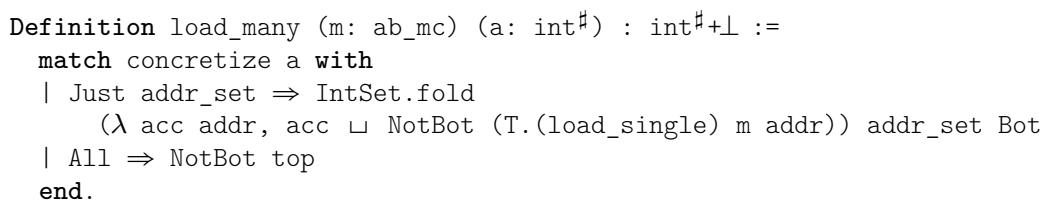

Figure 9 Example of abstract transformer

mem_dom) and its second occurrence is the concretization from the numeric domain ab_num.

Such an abstract memory domain is implemented using two maps. The first one, ab_reg, maps each register to an abstract numeric value and represents the register state. Thes second one, ab_mem, maps concrete addresses to abstract numeric values and represents the memory.

Record ab_machine_config :=

$\left\{\mathrm{ab} \_r e g: \operatorname{Map}\left[\mathrm{reg}\right.\right.$, int $\left.^{\sharp}\right] ;$ ab_mem: Map [ addr, int $\left.{ }^{\sharp}\right]$ \}.

To prevent the domain of the ab_mem map from infinitely growing, we bound it by a finite set computed before the analysis: the analysis will try to compute some information only for the memory addresses found in this set. The content of this set does not alter its soundness: the values stored at addresses that are not in it are unknown and the analyzer makes no assumptions about them. On the other hand, the success of the analysis and its precision depend on it. In particular, the analyzed set must cover the whole code segment. To compute this set, one possible method [1] is to start from an initial guess and, every time the analysis discovers that the set is too small (when it infers that control may reach a point that is not is the set), the analysis is restarted using a larger set. In practice, for all our examples, running the analysis once was enough, taking as initial guess the addresses of the instructions of the initial program.

\subsection{Abstract Semantics}

As a second layer, we build abstract transformers over any such abstract domain. Consider for instance the abstract load called load_many and presented in Figure 9; it is used to analyze any ILoad instruction ( $\mathrm{T}$ denotes a record of type mem_dom int $\sharp$ ab_mc). The source address may not be exactly known, but only represented by an abstract numeric value a. Since any address in $\gamma$ (a) may be read, we have to query all of them and take the least upper bound of all values that may be stored at any of these addresses: $\bigsqcup\{$ T. (load_single) $\mathrm{m} x \mid \mathrm{x} \in \gamma(\mathrm{a})\}$. However the set of concrete addresses may be huge and care must be taken: if the size of this set exceeds some threshold, the analysis gives up on this load and yields top, representing all possible values.

We build enough such abstract transformers to be able to analyze any instruction (function ab_post_single, shown in Figure 10). This function returns a list of possible next states, each of which being either Hlt $\mathrm{v}$ (the program halts 


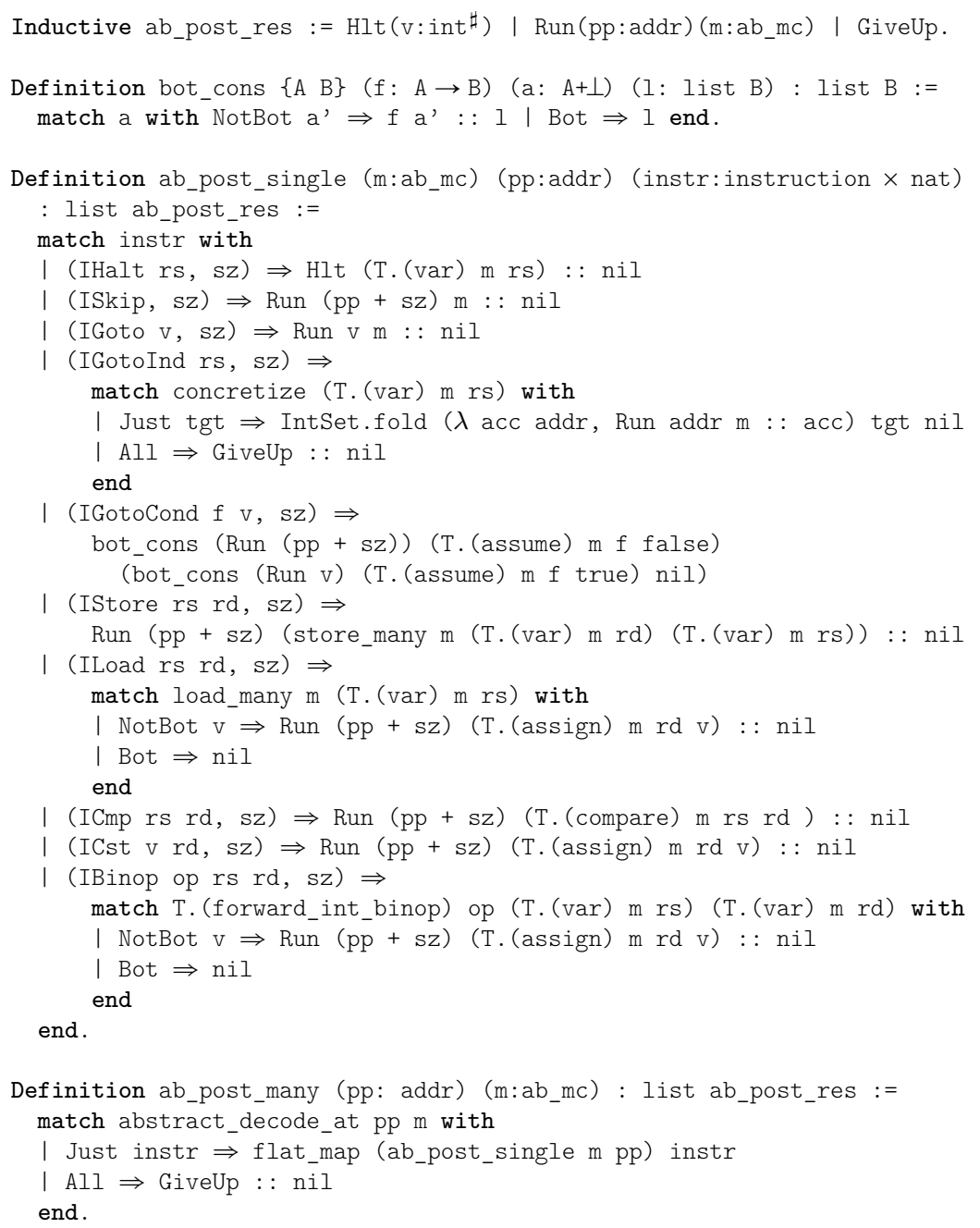

Figure 10 Abstract small-step semantics

returning a value approximated by $\mathrm{v}$ ) or Run $\mathrm{pp} \mathrm{m}$ (the execution proceeds at program point $\mathrm{pp}$ in a configuration approximated by $\mathrm{m}$ ) or GiveUp (the analysis is too imprecise to compute anything meaningful).

The computed jump (IGotoInd) also has a dedicated abstract transformer (inlined in Figure 10): in order to know from where to continue the analysis, we have to enumerate all possible targets. The abstract transformer for the conditional jump IGotoCond $\mathrm{f} v \mathrm{v}$ returns a two-element list. The first element means that the execution may proceed at $\mathrm{pp}+\mathrm{sz}$ (i.e., falls through) in a state where the branching flag $f$ is known to evaluate to false; the second element represents the case when the branch is taken: the flag is known to evaluate to 


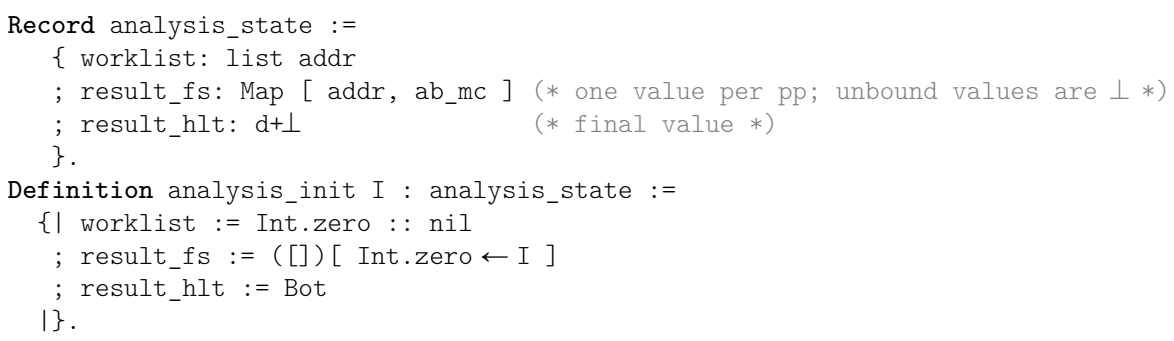

Figure 11 Internal state of the analyzer

true, and the next program point, v, is the one given in the instruction. Since each assume may return $\perp$ meaning that the considered branch cannot be taken, we use the combinator bot_cons that propagates this information: the returned list does not contain the unreachable states.

Then, function ab_post_many performs one execution step in the abstract. To do so, we first need to identify what is the next instruction, i.e., to decode in the abstract memory from the current program point. This may require to enumerate all concrete values that may be stored at this address. Therefore this abstract decoding either returns a set of possible next instructions or gives up. In such a case, the whole analysis will abort since the analyzed program is unknown.

\subsection{Fixpoint Computation}

Finally, a full program analysis is performed applying this abstract semantics iteratively. The analysis follows a worklist algorithm as the one found in [1, $\S 3.4]$. It maintains a state holding three pieces of data (see Figure 11):

1. the worklist, a list of program points left to explore; initially a singleton;

2. the current solution, mapping to each program point an abstract machine configuration; initially empty, but at program point zero, where it holds an abstraction of the program;

3. an abstraction of the final value, initially $\perp$.

A single step of analysis is performed by the function analysis_step shown in Figure 12. It picks a node $\mathrm{n}$ in the worklist - unless it is empty, meaning that the analysis is over - and retrieves the abstract configuration ab_mc associated with this program point in the current state. The abstract semantics is then applied to this configuration; it yields a list next of outcomes (see Figure 10) that are then propagated to the analysis state (function propagate). If the outcome is GiveUp, then the whole analysis aborts. Otherwise, if it is Run n' ab - meaning that ab describes reachable configurations at program point n' this abstract configuration is joined with the one previously associated with that program point. In case something new is learned, the program point n' is 


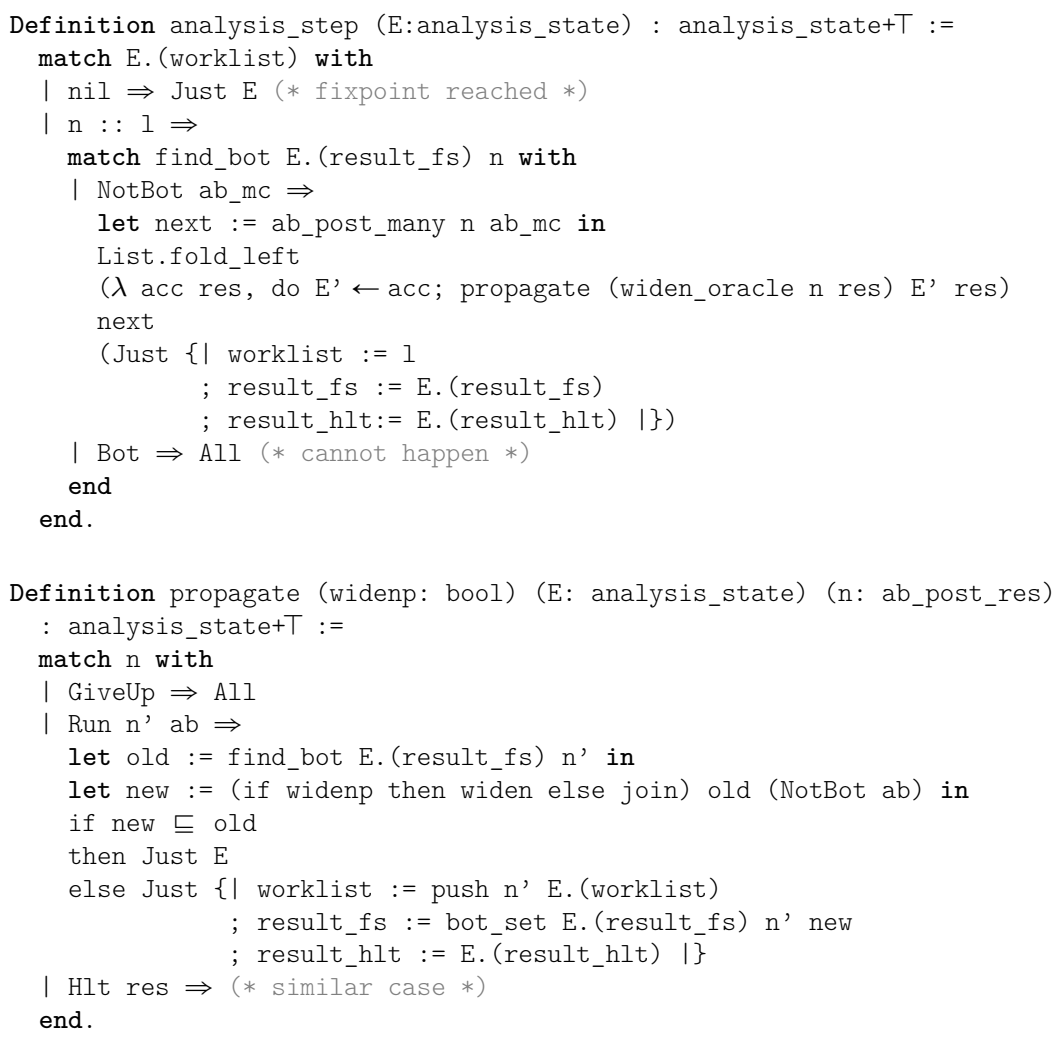

Figure 12 Body of the main analysis loop

pushed on the worklist. If it is Hlt res, then the abstraction of the final value is updated similarly.

Since there may be infinite ascending chains, so as to ensure termination, we need to apply widening operators instead of regular joins frequently enough during the search. Therefore the analysis is parameterized by a widening strategy that decides along which edges of the control-flow graph widening should be applied instead of a plain join. The implementation allows to easily try different strategies. The one we implemented mandates a widening on every edge from a program point to a smaller one, i.e., when jumping to a program point that is before in the address space.

The analysis repeatedly applies the analysis step until the worklist is empty (see Figure 13). So as to ensure that the analysis indeed terminates, we rely on a counter (known as fuel) that obviously decreases at each iteration; when it reaches zero, the analyzer must give up.

To enhance the precision, we have introduced three more techniques: a dedicated domain to abstract the flag state, a partitioning of the state space, 


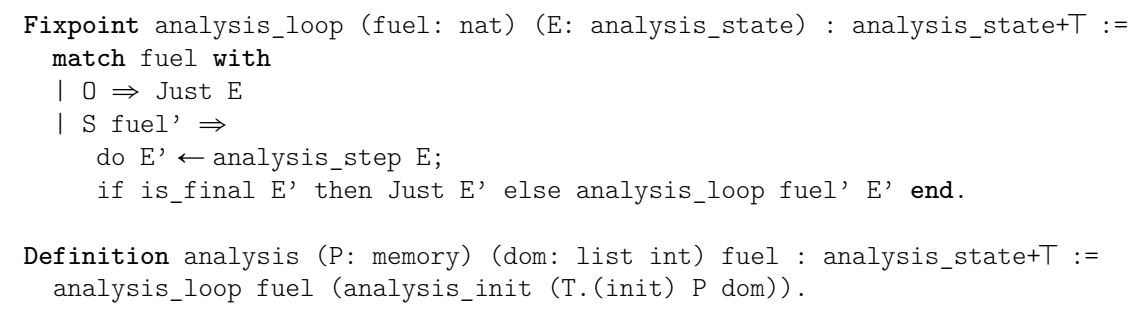

Figure 13 Main analysis

and a use of abstract instructions. They will be described in the next section $(\S 5)$; we first describe how we conducted the soundness proof of this analyzer.

\subsection{Soundness of the Abstract Interpreter}

We now describe the formal verification of our analyzer. The soundness property we ensure is that the result of the analysis of a program P over-approximates its semantics $\llbracket \mathrm{P} \rrbracket$. This involves on one hand a proof that the analysis result is indeed a fixpoint of the abstract semantics and on the other hand a proof that the abstract semantics is correct with respect to the concrete one.

The soundness of the abstract semantics is expressed by the following lemma, which reads: given an abstract state ab and a concrete one $\mathrm{m}$ in the concretization of $a b$, for each concrete small-step $m \leadsto m^{\prime}$, there exists a result $a b$ ' in the list ab_post_single m. (pc) ab that over-approximates m'. Our use of Coq type classes enables us to extensively overload the $\gamma$ notation and write this statement in a concise way as follows.

Lemma ab_post_many_correct : $\forall$ (m:machine_config) (m':machine_state) (ab:ab_mc), $\mathrm{m} \in \gamma(\mathrm{ab}) \rightarrow \mathrm{m} \leadsto \mathrm{m}^{\prime} \rightarrow \mathrm{m}^{\prime} \in \gamma($ ab_post_single m. (pc) ab).

The proof of this lemma follows from the soundness of the various abstract domains (as load_sound in Figure 8), transformers and decoder.

Lemma abstract_decode_at_sound : $\forall$ (m:machine_config) (ab:ab_mc) (pp:addr),

$\mathrm{m} \in \gamma(\mathrm{ab}) \rightarrow$ dec $\mathrm{m}$. (mc_mem) pp $\in \gamma($ abstract_decode_at pp ab).

The proof that the analyzer produces a fixpoint is not done directly. Instead, we rely on a posteriori verification: we do not trust the fixpoint computation and instead program and prove a checker called validate_fixpoint. Its specification, proved thanks to the previous lemma, reads as follows.

Lemma validate correct : $\forall$ ( $\mathrm{P}:$ memory) (dom: list addr) (E: AbEnv), validate_fixpoint $\mathrm{P}$ dom $\mathrm{E}=$ true $\rightarrow \llbracket \mathrm{P} \rrbracket \subseteq \gamma(\mathrm{E})$.

Going through this additional programming effort has various benefits. On the one hand, a direct proof of the fixpoint iterator would be very hard: in particular, it would require difficult proofs over the widening operators of all abstract domains [29]. On the other hand, we can adapt the iteration strategy, optimize the algorithm and so on with no additional proof effort. 
This validation checks two properties of the result $E$ : that the result overapproximates the initial state; and that the result is a post-fixpoint of the abstract semantics, i.e., for each abstract state in the result, performing one abstract step leads to abstract states that are already included in the result. These properties, combined to the soundness of the abstract semantics, ensure the conclusion of this lemma.

Finally we pack together the iterator and the checker with another operation performed on sound results that checks for its safety. The resulting analysis enjoys the following property: if, given a program $\mathrm{P}$, it outputs some result, then that program is safe.

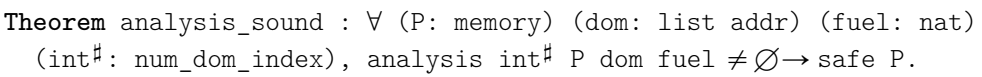

The arguments of the analysis program are the program to analyze, the list of addresses in memory to track, the counter that enforces termination and the name of the numeric domain to use. We provide two numeric domains: intervals with congruence information and finite sets.

\section{Case Studies and Analysis Extensions}

The extraction mechanism of Coq enables us to generate an OCaml program from our development and to link it with a front-end. Hence we can automatically analyze programs and prove them safe. This section shows the behavior of our analyzer on chosen examples, most of them taken from [11] (they have been rewritten to fit our custom syntax). All examples are written in an assembly-like syntax with some syntactic sugar: labels refer to byte offsets in the encoded program, the enc(i) notation denotes the encoding of the instruction i. The study of some examples highlights the limits of the basic technique presented before and drives some refinement of the analyzer as we describe below. These extensions have been integrated to our formalization and proved correct. The running time of the analysis of these examples is too short to be interesting to measure. The source code of all the examples that are mentioned thereafter is available on the companion web site [14].

\subsection{Basic Example}

The multilevel run-time code generation program of Figure 14 is a program that, when executed, writes some code to the addresses starting at line gen and runs it; this generated program, in turn, writes some more code at line ggen and runs it. Finally execution starts again from the beginning. Moreover, at each iteration, register $\mathrm{R} 6$ is incremented.

The analysis of such a program follows its concrete execution and exactly computes the content of each register at each program point. It thus correctly tracks what values are written and where, so as to be able to analyze the program as it is generated. 


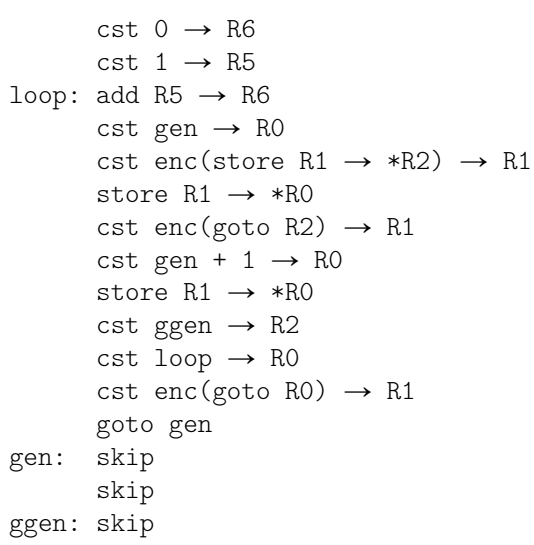

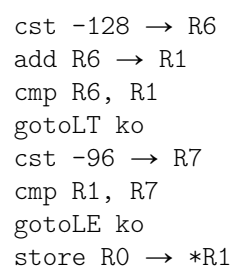

Figure 15 Array bounds check

Figure 14 Multilevel run-time code generation

However, when the execution reaches program point loop again, both states that may lead to that program point are merged. And the analysis of the loop body starts again. After the first iteration, the program text is exactly known, but each iteration yields more information about the dynamic content of register R6. Therefore we apply widening steps to ensure the termination of the analysis: the widening operator (of the memory domain) is used instead of the join operator on every edge from a program point to a smaller program point (i.e., in this example, from program point ggen to program point loop). Finally, the set of reachable program points is exactly computed and for each of them, we know what instruction will be executed from there.

Many self-modifying programs are successfully analyzed in a similar way: opcode modification, code obfuscation, and code checking [14].

\subsection{A First Extension: Dealing with Flags}

The example program in Figure 15 illustrates how conditional branching relies on implicit flags. This program stores the content of Ro in an array (stored in memory from address -128 to address -96 ) at the offset given in register R1. Before that store, checks are performed to ensure that the provided offset lies inside the bounds of the array. The destination address is compared against the lowest and highest addresses of the array; if any of the comparisons fails, then the store is bypassed.

To properly analyze this program, we need to understand that the store does not alter the code. When analyzing a conditional branch instruction, the abstract state is refined differently at its two targets, to take into account that a particular branch has been taken and not the other. However, the only information we have is about one flag, whereas the comparison that set this flag operated on the content of registers. We therefore need to keep the link between the flags and the registers. 
To this end, we extend our ab_machine_config record ${ }^{4}$ with a field containing an optional pair of registers ab_reg: $(r e g \times r e g)+T$. It enables the analyzer to remember which registers were involved in the last comparison (the $T$ value is used when this information is unknown). With such information available, even though the conditional jump is not directly linked to the comparison operation, we can gain some precision in the various branches. More precisely, the compare operator can now be implemented as follows.

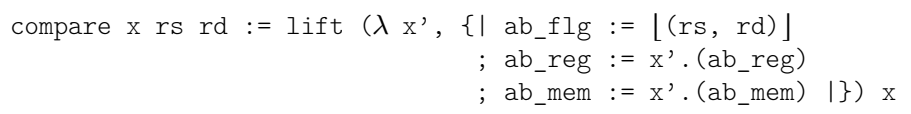

Back to the example of Figure 15, when we assume that the first conditional branch is not taken, the flag state is abstracted by the pair $[(R 6, R 1)\rfloor$, so we refine our knowledge about register R1: its content is not less than the content of register R6, namely -128 . Similarly, when we assume that the second conditional branch is not taken, the abstract flag state is $\lfloor(R 1, R 7)\rfloor$, so we can finally infer that the content of register $\mathrm{R} 1$ is in the bounds.

The actual implementation of such a precise assume relies on a backward transfer function of the numeric domain. The signature presented Figure 7 is extended with the two following fields: an abstract backward binary operation and its soundness criterion.

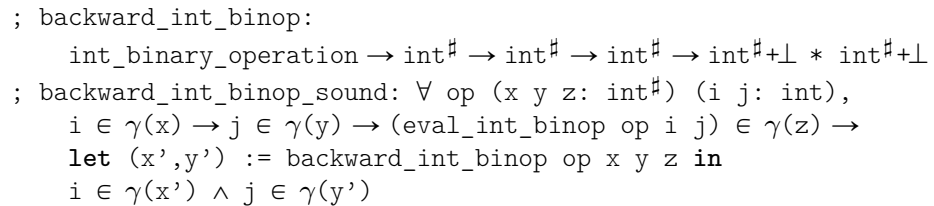

This transfer function takes as argument a binary operator op and three abstract values $\mathrm{x}, \mathrm{y}$ and $\mathrm{z}$, where $\mathrm{z}$ is meant to represent the result of the binary operation applied to $\mathrm{x}$ and $\mathrm{y}$ (or more precisely, to any values $\mathrm{i}$ and $j$ in their concretizations). It then returns a pair ( $\left.x^{\prime}, y^{\prime}\right)$ expected to be a more precise abstraction of the actual arguments. As an example, consider an interval domain. The call backward_int_binop OpAdd [0;4] [0;2] [5;6] could return the intervals $([3 ; 4],[1 ; 2])$ : knowing that the result of the addition is in the range $[5 ; 6]$ enables us to infer more precise ranges for the inputs.

Given such a backward transfer function, assume can be implemented as shown in Figure 16: if the registers Ru and Rv involved in the last comparison are known, then the abstract values $u$ and $v$ associated to them can be refined using the backward operator for the given comparison. In case any of these refined values is $\perp$, this information is propagated to the whole abstract state: the branch is unreachable and should not be analyzed any further.

Special care has to be taken in the assign transfer function. If a register that is part of the abstract flag is updated, then no information about its new content can be inferred from the outcome of the comparison. Therefore, in such cases, the abstract flag is simply forgotten, i.e., set to $T$.

4 This record has been introduced in Section 4.1. 


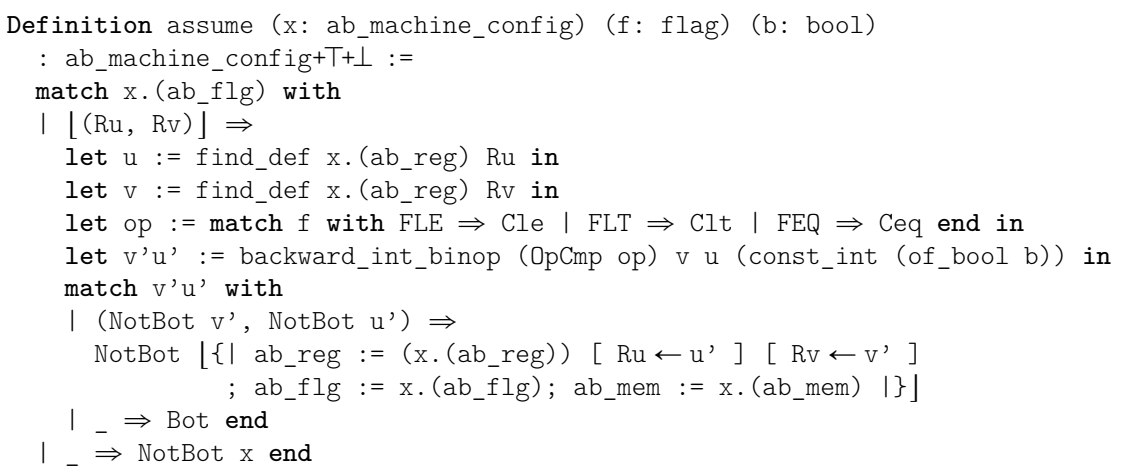

Figure 16 Implementation of the assume transfer function

This extension of the abstract domain has little impact on the formalization, but greatly increases the precision of the analyzer on programs with conditional branches. Indeed, without this extension, the analyzer cannot deduce anything from the guards of conditional branches as it ignores all comparison instructions.

\subsection{A Second Extension: Trace Partitioning}

During the execution of a self-modifying program, a given part of the memory may contain completely unrelated code fragments. When these fragments are analyzed, since they are stored at the same addresses, flow sensitivity is not enough to distinguish them. If these fragments are merged in the abstract state, then the two programs get mixed and it is no longer possible to predict the code that is executed with sufficient precision. To prevent such a precision loss, we use a specific form of trace partitioning [22] that makes an analysis sensitive to the value of a particular memory location.

Consider as an example the polymorphic program of Figure 17. Polymorphism here refers to a technique used by for instance viruses that change their code while preserving their behavior, so as to hide their presence. The main loop of this program (bytes 4 to 39) repeatedly adds forty-two to register R3 (two add instructions at bytes 13 and 14). However, it is obfuscated in two ways. First, the source code initially contains a jump to some random address (byte 13). But this instruction will be overwritten (bytes 7 to 12) before it is executed. Second, this bad instruction is written back (bytes 4 to 6 and 15 to 17), but at a different address (byte 14 is overwritten). The remainder of the loop swaps the contents of memory at addresses 11 and 16, and at addresses 12 and 17 (execution from byte 18 to byte 27 , and from byte 28 to byte 37 , respectively). So when the execution reaches the beginning of the loop, the program stored in memory is one of two different versions, both featuring the unsafe jump. In other words, this program features two variants that are functionally equivalent 


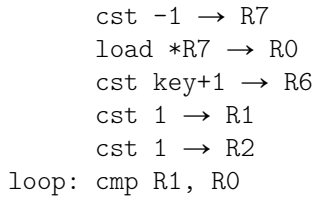

add $\mathrm{R} 4 \rightarrow \mathrm{R} 2$

store $\mathrm{R} 3 \rightarrow * \mathrm{R} 6$

goto loop

last: halt R2

Figure 18 Fibonacci

safety checker does not need to be updated since we can forget the partitioning before applying the original safety check.

Thanks to this technique, we can selectively enhance the precision of the analysis and correctly handle challenging self-modifying programs: control-flow modification, mutual modification, and code encryption [14]. However, the analyst must manually pick a suitable criterion for each program to analyze; the analyzer itself is not able to figure out what criterion to use. In practice, we have used the contents of some particular register or memory location.

When using this extension, the termination of the analysis may not be guaranteed any longer as the type $\mathrm{k}$ may have infinitely many values (or too many for the analysis to enumerate them all). To ensure termination, Kinder [22] proposes a widening operator that merges keys at a particular program point when the number of different keys encountered at this program point exceeds some threshold. We did not implement such a widening operator and require the analyst to be careful when the partitioning criterion is designed.

\subsection{A Third Extension: Abstract Decoding}

The program in Figure 18 computes the $n^{\text {th }}$ Fibonacci number in register R2, where $n$ is an input value read from address -1 and held in register Ro. There is a for-loop in which register R1 goes from 1 to $n$ and some constant value is added to register R2. The trick is that the actual constant (which is encoded as part of an instruction and is stored at the address held in R6) is overwritten at each iteration by the previous value of R2.

When analyzing this program, we cannot infer much information about the content of the patched cell. Therefore, we cannot enumerate all instructions that may be stored at the patched point. So we introduce abstract instructions: instructions that are not exactly known, but of which some part is abstracted by a suitable abstract domain. Here we only need to abstract values using a numeric domain: the resulting instruction set is shown in Figure 19. This abstraction of the instructions could be pushed further to capture other selfmodification patterns. For instance a program might modify only the encoding of a register; in such a case, the "register" part of the instructions could be abstracted by a finite set of registers.

With such a tool, we can decode in the abstract: the analyzer does not recover the exact instructions of the program, but only the information that 


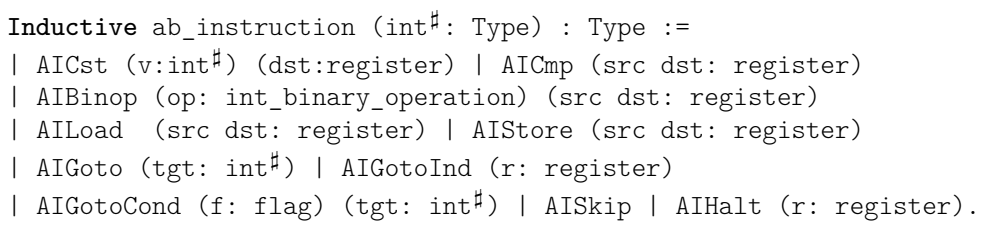

Figure 19 Abstract instructions

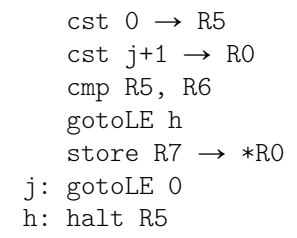

Figure 20 Not-a-branch

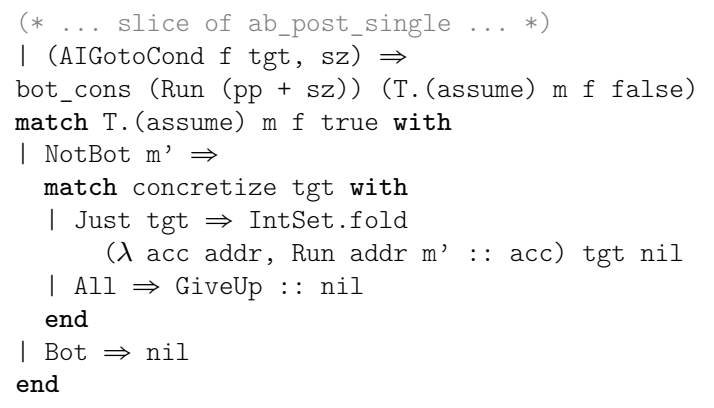

Figure 21 Abstract conditional jump

some (unknown) value is loaded into register R4, which is harmless (no stores and no jumps depend on it).

This self-modifying code pattern, in which only part of an instruction is overwritten occurs also in the vector dot product example [14] where specialized multiplication instructions are emitted depending on an input vector.

For this technique to be effective, the numeric abstract domain has to support it: mapping abstract values to abstract instructions (i.e., abstract decoding) should be more efficient than just enumerating all concrete values.

The abstract semantics (Figure 10) has to be slightly modified to deal with this new instruction set. In particular, all jumps behave like indirect jumps: their targets are only known as abstract values. For the analysis to follow such a jump, all concrete destinations need to be enumerated. However, consider the example shown Figure 20: it compares an input value (held in register R6) to zero, and depending on the outcome, either terminates, modifies itself and then terminates. The conditional jump on line $j$ is unsafe: its target is overwritten on the line before with some input value (the contents of register R7). But the whole program is actually safe, because the branching condition is always false $^{5}$. Therefore, the abstract transformer for conditional jumps (Figure 21) tries to prove that the branch cannot be taken before it enumerates its possible targets; and the program of Figure 20 can be proved safe by our analyzer.

\footnotetext{
5 Such spurious branching instructions are known as "opaque predicates" and used mainly for obfuscation [13].
} 


\begin{tabular}{lccc}
\hline \multicolumn{1}{c}{ Program } & Result & Comment \\
\hline Opcode modification & $\checkmark$ & \\
Multi-level run-time code gen. & $\checkmark$ & \\
Bootloader & $\times$ & needs a model of system calls and interrupts \\
Control-flow modification & $\checkmark$ & partitioning on the jump target (address 15) \\
Vector dot product & $\checkmark$ & partitioning on loop counter (register R0); and \\
Run-time code checking & $\checkmark$ & & abstract decoding \\
Fibonacci & $\checkmark$ & abstract decoding \\
Self-replication & $\times$ & code segment is "infinite" \\
Mutual modification & $\checkmark$ & partitioning on the instruction to write (held \\
Polymorphic code & & in register R0) \\
Code obfuscation & $\checkmark$ & partitioning according to the different versions \\
Code encryption & $\checkmark$ & \\
\hline
\end{tabular}

Figure 22 Summary of self-modifying examples

\subsection{Summary of the Case Studies}

The techniques presented here enable us to automatically prove the safety of various self-modifying programs including almost all the examples of Cai et al. [11] as summarized in Figure 22. Out of twelve, only two cannot be dealt with. The comment column of the table lists the extensions that are needed to handle each example (if any), or the limitation of our analyzer. The boot loader example does not fit in the considered machine model, as it calls BIOS interrupts and reads files. The self-replicating example is a program that fills the memory with copies of itself: the code, being infinite, cannot be represented with our abstract domain. Our Coq development [14] features all the extensions along with their correctness proofs, and several example programs including the implementation of the programs listed in Figure 22.

\section{Related Work}

Most of the previous works on mechanized verification of static analyzes focused on standard data-flow frameworks $[23,15,4,10]$ or abstract interpretation for small imperative structured languages $[3,8,27]$. Klein and Nipkow instantiate such a framework for inference of Java bytecode types [23]; Coupet-Grimal and Delobel [15] and Bertot et al. [4] for compiler optimizations, and Cachera et al. [10] for data-flow analysis.

The first attempt to mechanize abstract interpretation in its full generality is Monniaux's master's thesis [24]. Using the Coq proof assistant and following the orthodox approach based on Galois connections, he runs into difficulties with $\alpha$ abstraction functions being nonconstructive, and with the calculation of abstract operators being poorly supported by Coq. Later, Pichardie's Ph.D. thesis [30, $28]$ mechanizes the $\gamma$-only presentation of abstract interpretation that we use. 
Blazy et al. use Pichardie's approach to verify an interval analysis for the RTL intermediate language of the CompCert formally verified compiler [6]. Our current work shares the same notion of abstract numerical domain but develops its own notion of memory abstraction, dynamic control-flow graph reconstruction and trace partitioning. Bertot [3] and Nipkow [27] give alternate presentations of Pichardie's approach, respectively in Coq and in Isabelle/HOL, resulting in pedagogical abstract interpreters for the IMP mini-language.

Jourdan et al. extend the work described in [6] to a much more complex source language, most of the ISO C 1999 language (excluding recursion and dynamic allocation) [20] and use a more sophisticated static analysis technique (combination of several abstract domains, including relational domains). Our current work formalizes complementary advanced abstract interpretation techniques, but targeting self-modifying low-level code, and is based on several recent non-verified static analyses. A large amount of work was done by Balakrishnan et al. in this area [1]. Control-flow graph reconstruction was specially studied by Kinder et al. [22] and Bardin et al. [2]. Still, these works are unsound with respect to self-modifying code: they assume that the code cannot be modified by memory writes and do not check that this assumption holds for the analysed programs. Therefore, the analysis of self-modifying programs can yield incorrect results.

The first formal semantics for self-modifying programs are defined in [18], and then in [17] and [7]. These are paper-and-pencil semantics that operate over small low-level languages as ours; they are used in a very different way from our semantics. Gerth [18] defines an axiomatics semantics where semantic states are modeled as arrays indexed by an instruction pointer. In [17], a denotational semantics based on traces is used to identify the different phases of successive program modifications during a program execution. An operational semantics is defined in [7], where a rewriting process from self-modifying programs to non-modifying programs is defined.

Our current work tackles a core subset of a self-modifying low-level programming language. More realistic formalizations of x86 semantics were proposed [26, $25,21]$ but none of them handles the problem of disassembling self-modifying programs. Our work complements other verification efforts of low-level programs $[12,11,19]$ based on program logics. While we provide automatic inference of loop invariants, they are able to handle more expressive correctness properties.

\section{Conclusion and Perspectives}

This work provides the first verified static analysis for self-modifying programs. In order to tackle this challenge, we formalized original techniques such as control-flow graph reconstruction and partitioning. We formalized these techniques on a small core language but we managed to verify ten out of twelve of the challenges proposed in [11]. 
An important further work is to scale these technique to more realistic Coq language models $[25,21]$. Developing directly an analyzer on these representations may be a huge development task because of the number of instructions to handle. One strategy could be to relate on a good intermediate representation such as the one proposed by Rocksalt [25]. Our current work does not consider the specific challenge of call stack reconstruction [1] that may require some form of verified alias analysis [31]. This is an important place for further work.

\section{References}

[1] Gogul Balakrishnan and Thomas W. Reps. "WYSINWYX: What you see is not what you eXecute." In: ACM Trans. Program. On Lang. Syst. 32.6 (2010).

[2] Sébastien Bardin, Philippe Herrmann, and Franck Védrine. "RefinementBased CFG Reconstruction from Unstructured Programs." In: Verification, Model Checking and Abstract Interpretation (VMCAI). Vol. 6538. LNCS. Springer, 2011, pp. 54-69.

[3] Yves Bertot. "Structural abstract interpretation, A formal study in Coq." In: Language Engineering and Rigorous Software Development, International LerNet ALFA Summer School 2008. Vol. 5520. LNCS. Springer, 2009, pp. 153-194.

[4] Yves Bertot, Benjamin Grégoire, and Xavier Leroy. "A structured approach to proving compiler optimizations based on dataflow analysis." In: TYPES. Vol. 3839. LNCS. Springer, 2006, pp. 66-81.

[5] Sandrine Blazy and Xavier Leroy. "Mechanized semantics for the Clight subset of the C language." In: Journal of Automated Reasoning 43.3 (2009), pp. 263-288.

[6] Sandrine Blazy et al. "Formal Verification of a C Value Analysis Based on Abstract Interpretation." In: Static Analysis Symposium (SAS). Vol. 7935. LNCS. Springer, 2013, pp. 324-344.

[7] Guillaume Bonfante, Jean-Yves Marion, and D. Reynaud-Plantey. "A Computability Perspective on Self-Modifying Programs." In: SEFM. 2009, pp. 231-239.

[8] David Cachera and David Pichardie. "A Certified Denotational Abstract Interpreter." In: Interactive Theorem Proving (ITP). Vol. 6172. LNCS. Springer, 2010, pp. 9-24.

[9] David Cachera and David Pichardie. "Comparing Techniques for Certified Static Analysis." In: Proc. of the 1st NASA Formal Methods Symposium (NFM'09) (2009), pp. 111-115.

[10] David Cachera et al. "Extracting a data flow analyser in constructive logic." In: Theor. Comput. Sci. 342.1 (2005), pp. 56-78.

[11] Hongxu Cai, Zhong Shao, and Alexander Vaynberg. "Certified SelfModifying Code." In: Conf. on Programming Language Design and Implementation (PLDI). ACM, 2007, pp. 66-77. 
[12] Adam Chlipala. "Mostly-automated verification of low-level programs in computational separation logic." In: Conf. on Programming Language Design and Implementation (PLDI). ACM, 2011.

[13] Christian Collberg, Clark Thomborson, and Douglas Low. "Manufacturing cheap, resilient, and stealthy opaque constructs." In: 25th Symp. on Principles of Programm. Lang. (POPL). ACM, 1998, pp. 184-196.

[14] Companion website. URL: http://www.irisa.fr/celtique/ext/smc.

[15] Solange Coupet-Grimal and William Delobel. "A Uniform and Certified Approach for Two Static Analyses." In: TYPES. Vol. 3839. LNCS. 2004, pp. $115-137$.

[16] Patrick Cousot and Radhia Cousot. "Abstract interpretation: a unified lattice model for static analysis of programs by construction or approximation of fixpoints." In: Symp. on Principles of Programm. Lang. (POPL). ACM, 1977, pp. 238-252.

[17] Saumya K. Debray, Kevin P. Coogan, and Greg M. Townsend. "On the semantics of self-unpacking malware code." Draft. 2008.

[18] Rob Tristan Gerth. "Formal Verification of Self Modifying Code." In: Int. Conf. for Young Computer Scientists. International Academic Publishers, China, 1991.

[19] Jonas Jensen, Nick Benton, and Andrew Kennedy. "High-Level Separation Logic for Low-Level Code." In: Symp. on Principles of Programm. Lang. (POPL). ACM, 2013.

[20] Jacques-Henri Jourdan et al. "Formal verification of a C static analyzer." In: 42nd symposium Principles of Programming Languages (POPL). 2015, pp. 247-259.

[21] Andrew Kennedy et al. "Coq: The world's best macro assembler?" In: Symp. on Principles and Practice of Declarative Programming (PPDP). ACM, 2013, pp. 13-24.

[22] Johanes Kinder. "Towards static analysis of virtualization-obfuscated binaries." In: Working Conference on Reverse Engineering. 2012, pp. 6170.

[23] Gerwin Klein and Tobias Nipkow. "A Machine-Checked Model for a Java-Like Language, Virtual Machine and Compiler." In: ACM Trans. On Programm. Lang. And Syst. 28.4 (2006), pp. 619-695.

[24] David Monniaux. "Réalisation mécanisée d'interpréteurs abstraits." MA thesis. U. Paris 7, 1998.

[25] Greg Morrisett et al. "RockSalt: better, faster, stronger SFI for the x86." In: Conf. on Programming Language Design and Implementation (PLDI). 2012, pp. 395-404.

[26] Magnus O. Myreen. "Verified just-in-time compiler on x86." In: Symp. on Principles of Programm. Lang. (POPL). ACM, 2010, pp. 107-118.

[27] Tobias Nipkow. "Abstract Interpretation of Annotated Commands." In: Interactive Theorem Proving (ITP). Vol. 7406. LNCS. Springer, 2012, pp. 116-132. 
[28] David Pichardie. "Building Certified Static Analysers by Modular Construction of Well-founded Lattices." In: Electr. Notes Theor. Comput. Sci. 212 (2008), pp. 225-239.

[29] David Pichardie. "Building certified static analysers by modular construction of well-founded lattices." In: Proc. of the $1^{\text {st }}$ International Conference on Foundations of Informatics, Computing and Software (FICS). Vol. 212. Electronic Notes in Theoretical Computer Science. 2008, pp. 225-239.

[30] David Pichardie. "Interprétation abstraite en logique intuitionniste: extraction d'analyseurs Java certifiés." PhD thesis. U. Rennes 1, 2005.

[31] Valentin Robert and Xavier Leroy. "A Formally-Verified Alias Analysis." In: Certified Proofs and Programs (CPP). Vol. 7679. LNCS. Springer, 2012, pp. 11-26.

[32] Gordon Stewart, Lennart Beringer, and Andrew W. Appel. "Verified heap theorem prover by paramodulation." In: Int. Conf. on Functional Programming (ICFP). ACM, 2012, pp. 3-14.

[33] Peter Szor. The Art of Computer Virus Research and Defense. AddisonWesley Professional, 2005. ISBN: 0321304543. 